\title{
Análisis semiótico de videos en la resolución de problemas de la física
}

DOI: https://doi.org/10.33262/ap.v1i2.7

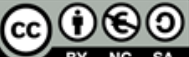

Semiotic video analysis in physics problem solving

Ariel León Arencibia., ${ }^{1}$ Bárbaro Evelio Rodríguez Brito. ${ }^{2}$ \& Beatriz de la Caridad Dorvigny González. ${ }^{3}$

\begin{abstract}
.
Today's world is characterized by electronic development in different social spheres. Cuba is not exempt from this situation, the country is going through a colossal battle of ideas, and the educational work of the Revolution and its transformations is irrefutable proof of this assertion. New schools, excellent study materials and the application of the new programs of the revolution put the teacher in a position to achieve results superior to all the previous work. The computer allows automating the processing of data and immediately presenting the results in graphical form and equations, its use in an experiment contributes to raising its quality, for other reasons related to fundamental objectives of the teaching-learning process of Science. Also the semiotic analysis on the text constituted by the simulation, from the Peirce triad, originates the phenomenon by which a sign gives birth to another sign and, especially, a thought gives birth to another thought. In this process of meanings can be seen as interrelated cultural units with each other, considering the different interpret involved in the teaching-learning process. The aim of the paper is to present how to make use of Tracker, IDES and GeoGebra software with a small description from the semiotic of its meaning, allowing the analysis of videos related to practical life.
\end{abstract}

\footnotetext{
${ }^{1}$ Universidad de La Habana. Facultad de Turismo. La Habana, Cuba. smfdariel@gmail.com

${ }^{2}$ Universidad de La Habana. Facultad de Turismo. La Habana, Cuba. smfdariel@gmail.com

${ }^{3}$ Universidad de La Habana. Facultad de Turismo. La Habana, Cuba. darielarmstrong@gmail.com
} 
Keywords: Semiotics, video analysis, model, tracker.

\section{Resumen.}

El mundo de hoy está caracterizado por el desarrollo electrónico en las diferentes esferas sociales. Cuba no está exenta a esta situación. Escuelas nuevas, excelentes materiales de estudios y la aplicación de los nuevos programas de la revolución ponen al maestro en condiciones para lograr resultados muy superiores a todo el quehacer precedente. La computadora permite automatizar el procesamiento de datos e inmediatamente presentar los resultados en forma gráfica y de ecuaciones, su empleo en un experimento contribuye a elevar su calidad, por otras razones relacionadas con objetivos fundamentales del proceso de enseñanza aprendizaje de la Ciencia. También el análisis semiótico sobre el texto constituido por la simulación, a partir de la triada de Peirce, origina el fenómeno por el cual un signo da nacimiento a otro signo y, especialmente, un pensamiento da nacimiento a otro pensamiento. En el trabajo se pretende presentar y ejemplificar cómo hacer uso del software Tracker, IDES y GeoGebra con una pequeña descripción desde la semiótica de su significado, permitiendo hacer el análisis de videos relacionados con la vida práctica en el curso Optativo de la Carrera de Licenciatura en Educación. Física, siendo el mismo una herramienta poderosa para el desarrollo del proceso enseñanza aprendizaje de la Física.

Palabras claves: Semiótica, análisis de videos, modelo, tracker.

\section{Introducción.}

El mundo de hoy está caracterizado por el desarrollo electrónico en las diferentes esferas sociales. Cuba no está exenta a esta situación, en tal sentido Guevara expresó: "Todo indica que esta ciencia se constituirá en algo así como una medida del desarrollo; quien la domine será un país de vanguardia. Vamos a volcar nuestros esfuerzos en este sentido con audacia revolucionaria" (Revista Bohemia, 1962, p.18)

El país transita por una colosal batalla de ideas, la obra educacional de la Revolución y sus transformaciones es una prueba irrefutable de esta aseveración. Escuelas en mejores condiciones, excelentes y modernos materiales de estudios ponen al maestro en condiciones para lograr resultados muy superiores a todo el quehacer precedente.

El Comandante en Jefe a propósito de estas transformaciones trazó pautas, cuando en su intervención el 16 de septiembre de 2004 señaló:

Hoy se trata de perfeccionar la obra realizada y partiendo de ideas y conceptos enteramente nuevos. Hoy buscamos lo que a nuestro juicio debe ser y será un sistema educacional que se corresponda cada vez más con la igualdad, la justicia plena, la autoestima, y las necesidades morales y sociales de los ciudadanos. En el modelo de sociedad que el pueblo de Cuba se ha propuesto crear (Castro, 2004, s/p.). 
Donde se hace necesario indagar en nuevas vías aprovechando al máximo los avances de la tecnología para poner en práctica los conocimientos que se les trasmiten a los estudiantes desde su formación en la carrera Matemática- Física en las Universidades Pedagógicas, así como lograr un mayor desarrollo de habilidades centrando su atención y el interés de lo estudiado.

Las TIC componen en la actualidad el soporte material del proceso de enseñanza aprendizaje. Ellos se encuentran en estrecha relación con los objetivos, el contenido y los métodos educativos. Estos medios desempeñan un papel fundamental en el proceso lógico de obtención del conocimiento, cuyas fases son de la realidad objetiva al pensar abstracto y de este a la práctica.

Permitiendo intensificar el proceso de enseñanza aprendizaje, porque con su utilización se logra que los estudiantes aprendan más, memoricen mejor y además una racionalización del tiempo necesario para el aprendizaje. En la medida en que se emplean medios más objetivos y concretos (más cercanos a la realidad), el tiempo necesario para aprender más y mejor, se acorta hasta en siete veces.

Con el uso de las mismas se pretende elevar la eficiencia del sistema educacional, garantizando una docencia de mayor calidad y con mejores resultados. Además, permiten disminuir los esfuerzos del profesor y del alumno proporcionando un mejor aprovechamiento de la fuerza laboral. Se logra que el aprendizaje se comporte de un modo ascendente y estructurado, de manera sistemática; de forma tal, que unos conocimientos se integran a los anteriores para proporcionar más elementos y mejores.

Contribuyen a hacer más duraderos los conocimientos adquiridos, sino que también aumentan la motivación por el aprendizaje y por la asignatura en particular. Este elemento motivacional se consigue, en parte, por la presentación de estímulos más vivos que facilitan la autoactividad del estudiante (como en los laboratorios o textos y su vinculación con los Software), la seguridad en el proceso del aprendizaje, o el cambio de actividad en la exposición rutinaria de las clases tradicionales.

Los estudiantes deben aprender a usar las nuevas tecnologías para enseñar y aprender respectivamente, para desempeñarse satisfactoriamente en la sociedad, jugando un papel activo en la solución de los problemas que atentan contra la humanidad. Pero no se debe perder de vista el estudio de los procesos interactivos que se dan en un ambiente de aprendizaje colaborativo mediado por computadora. Considerando los distintos interpretantes involucrados en el proceso de enseñanza aprendizaje, alumnos, docente, las herramientas informáticas y el análisis de videos relacionados con la vida práctica, por lo que se pretende realizar un estudio semiótico en el que se analiza el texto constituido por la simulación, a partir de la triada de Peirce. 
El estudio semiótico realizado, sobre el análisis de videos con el uso de Tracker, se constituye en una herramienta de análisis fundamental para que puedan darse las condiciones aceptadas, sirviendo de trampolín para el diseño de nuevas situaciones de enseñanza.

Cada vez que el hombre se enfrenta a una realidad, busca en los archivos de su cultura aquellos que le permitan comprender lo que el contexto le propone para interpretarlo. Generándose un diálogo entre cultura, contexto y cognición. La cultura le permite el encuentro con la memoria colectiva y el contexto funciona como elemento instrumental de las nuevas experiencias y también como agente de apropiación. Este proceso de interpretación origina el fenómeno por el cual un signo da nacimiento a otro signo y, especialmente, un pensamiento da nacimiento a otro pensamiento. En este proceso, los significados pueden verse como unidades culturales interrelacionadas unas con otras (Eco, 1986).

\section{Desarrollo.}

La semiótica peirceana como herramienta de análisis

Como rama de la filosofía la semiótica es una ciencia normativa, formal. Se refiere a los signos y a su valor de verdad. Apunta a la verdad, se puede expresar y conocer a partir de los signos, y sirve para establecer los principios rectores para cualquier otra ciencia que opere con signos. Se interesa por determinar las condiciones necesarias y esenciales del carácter y el empleo de los signos.

El filósofo americano Peirce, en Collected Papers, plantea un abordaje pragmático-cognitivo cercano a la lógica y a la fenomenología. Este enfoque cognitivo considera a la semiótica como la identificación de un conjunto de operaciones mentales.

La inclusión, en la propia estructura del signo, del interpretante, es uno de los rasgos de la actualidad de Peirce. Considera que un signo no es tal hasta que no es descifrado por el receptor e intérprete de éste y que no preexiste antes de que ocurra este hecho. La observación introduce nuevas ideas mientras que la inferencia combina estas ideas con otras para llegar a nuevas proposiciones. La inferencia tiene tres formas básicas: la abducción, la deducción y la inducción. (Magariños, 2007).

La semiótica comprende tres ramas: 1) el estudio de las condiciones necesarias que cuentan para que un signo sea tal: gramática semiótica; 2) el establecimiento de los criterios para considerar que algo es verdadero por medio de inferencias de y a través de los signos: lógica crítica, y 3) la determinación de las condiciones y el desarrollo de los signos: retórica universal.

El signo según Peirce. 
Un signo, o representamen, es algo que está para alguien, por algo, en algún aspecto o disposición:

Ello se dirige a alguien, o sea, crea en la mente de esa persona un signo equivalente, o quizá un signo más desarrollado. A este que aquél crea, lo denominó el interpretante del primer signo. El signo está por algo, su objeto. Este está por tal objeto, no en todo sentido, sino respecto a un tipo de idea que algunas veces he llamado el fundamento del representamen. (Magariños, 2007, p. 64)

El proceso del conocimiento es también una relación entre representamen e interpretante; el representamen es el concepto o enunciado, por ejemplo científico, que se dirige a un sistema teórico donde transformado en interpretante, o sea en un lugar lógico, recibe su valor significativo, el cual, posiblemente, incrementa el que tenía en cuanto propuesta o representamen (Magariños, 1983).

Peirce constituye al signo como capaz de sustituir a su objeto, del que solo puede decir que es algo. Esta sustitución no lo es en cuanto su totalidad, sino respecto a una parte de su posibilidad sustitutiva, a un tipo de idea, a la que denomina fundamento (Magariños, 1983). El signo tiene como objeto siempre a otro signo. Es decir, no existe signo que no sea reducible a otro signo. Esta oclusión del signo, tanto en lo que respecta al interpretante, atribuyéndole el carácter de signo, como en lo que respecta al fundamento, estableciéndolo igualmente como signo, implica una recursividad en la estructura de la gramática de los signos a la que Peirce denomina Gramática especulativa (Magariños,1983).

Así, el conocimiento tiene siempre por objeto a otro conocimiento y nunca a la realidad en su pretendida pureza de no modificada todavía por el pensamiento. El objeto de todo signo debe ser algo ya conocido, que también es signo, entonces el signo único es incognoscible. Ninguno de los tres componentes del signo, ni el fundamento, ni el representamen, ni el interpretante, tienen sentido por sí solos. (Magariños, 1983).

El signo se genera en un ámbito semiótico que debe ser acotado y ajustado lógicamente para que pueda reconocerse como tal. Los tres aspectos que requiere la existencia del signo son: el "por algo", el "para alguien" y el "en alguna relación". Mediante el primero, el signo captará lo que de conocimiento (fundamento) le interesa del objeto; mediante el segundo, se instituirá a sí mismo como forma perceptual y soporte sustitutivo (representamen) de tal intervención; $\mathrm{y}$, mediante el tercero, proporcionará la posibilidad de modificación que, en un determinado sistema (interpretante), afecta al conocimiento o desconocimiento (pero no, noconocimiento) acerca de dicho objeto. Lo que hace alusión a los conceptos de primeridad, segundidad y terceridad propuestos por Peirce.

Cada signo para ser tal debe ser interpretado, es decir debe ser capaz de determinar un interpretante. 
Este será el producto del proceso de semiosis, es otro signo que resulta de signos anteriores y se desarrollan a través de la mediación de estos. Los interpretantes se dividen en: inmediato (interpretante tal como es representado o significado en el signo. Por ejemplo: vagas impresiones, cualidades, la idea de un esfuerzo o la idea de un tipo general), dinámico (es el efecto directo o actual producido por un signo en una acción interpretativa. Por ejemplo: acciones, hechos o ideas producidos singularmente), es el sentimiento que produce) y final (efecto de cualquier regla o ley que un signo tiene sobre la acción interpretativa. Por ejemplo: las leyes, los hábitos, las disposiciones y las regularidades)

El interpretante final puede verse como el medio por el cual un signo se traduce en otro sistema de signos; o verse como el estado de información que el signo brinda (proceso de traducción, como producto); también puede verse como las reglas de tales traducciones, que para Peirce son los distintos tipos de inferencia o razonamiento: la abducción, la inducción y la deducción, interrelación triádica del signo, objeto e interpretante da cuenta de la acción del signo, o sea de la semiosis, a la que Peirce define como un acto o influencia que es o envuelve una cooperación de tres sujetos, tales como un signo, su objeto y su interpretante, relación de influencia que no es de ninguna manera resoluble en actos entre pares.

Así cada signo puede analizarse en tres aspectos que se corresponden con las primeras tres condiciones formales del signo:

- El signo cual signo (carácter presentativo, cualidad abstracta o forma de un signo). Se clasifica en: Cualisigno cuando el carácter presentativo comprende sus características cualitativas, Sinsigno: el carácter presentativo del signo se da en términos de sus cualidades existenciales; y Legisigno: el carácter presentativo del signo se da a partir de cualquier forma convencional, de disposición o legitimidad que pueda haber adquirido.

- El signo en relación con su objeto (carácter representativo, el objeto como determinante del signo y el signo como representación del objeto). Se clasifica en Icono: cuando las características presentativas del signo son similares a las del objeto ( si comparten cualidades simples son imágenes, los que representan las relaciones de las partes por medio de relaciones análogas son los diagramas y cuando su carácter representativo está representado por un paralelo con el carácter representativo de otro signo se denominan metáforas); Índice: cuando representan a su objeto por medio de contigüidad (cuando hay referencia directa son designativos, pueden ser causales o reagentes cuando son provocados por el objeto que representan); Símbolo: representan a su objeto por medio de alguna relación convencional, habitual, disposicional o cualquier otra relación de tipo legal (puede ser genuino, cuando el objeto al que se refiere es usualmente general, singular si el objeto es un existente individual y abstracto si el objeto es algún carácter o cualidad).

- El signo en relación con su interpretante (carácter interpretativo, inmediato, dinámico y final). Se clasifica en: Rema: orienta al interpretante sobre las características interpretativas del signo (un término); Dicisigno: conecta el sentido con el referente (la proposición); y Argumento: signo cuya interpretación está dirigida a una conexión sistemática, inferencial y legal con otros signos (argumento silogístico). 
Los signos y los símbolos constituyen un sistema de comunicación convencionalmente distinguidos de los otros sistemas de comunicación. Se presentan como un reticulado textual cuyos elementos tienen su propia función interpretativa.

El análisis semiótico de videos en ambientes educativos.

El autor sugiere que el ejemplo más simple de interacción con las máquinas digitales esconde una intrincada red de procesos semióticos y cognitivos que pueden estudiarse (Scolari, 2003, p. 1). Este autor propone la elaboración de un modelo sociosemiótico que permitan comprender mejor la interacción persona-ordenador, sosteniendo que las interfaces interactivas que pueden presentar las herramientas informáticas, actúan con una gramática interactiva propia que dialoga con los otros sistemas de interacción (social y cognitivo) y se interrelacionan y resignifican mutuamente El autor sugiere que el ejemplo más simple de interacción con las máquinas digitales esconde una intrincada red de procesos semióticos y cognitivos que pueden estudiarse (Scolari, 2003). La interfaz de una herramienta interactiva, como cualquier otro lugar donde se verifican procesos semióticos, nunca es neutral ni ingenua y la interacción con ellas está lejos de ser una actividad automática, natural y transparente (Scolari, 2004).

La interacción de un sujeto con la computadora no es solo una acción natural y automática. (Scolari, 2003) Antes, durante y después de la acción es posible identificar procesos perceptivos de reconocimiento, intercambios comunicativos a nivel textual entre emisor y receptor, que remiten a experiencias precedentes de interacción. En el análisis videos con tracker, en las fases de diseño como durante la interacción con las herramientas se ponen en juego procesos y competencias semióticas.

Siendo una herramienta que invita a la interacción, atrae al usuario a partir de una gramática interactiva propia, a partir de la creación de mecanismos autónomos que nada tienen que ver con los objetos del mundo material.

La Física utiliza modelos matemáticos que permiten la elaboración de modelos con la computadora y el aprovechamiento de videos, posibilitando la creación de representaciones figurativas que permiten la manipulación de objetos asociados a conceptos con alto grado de abstracción.

Desde el punto de vista epistemológico, un modelo es una entidad no lingüística que sirve al individuo a modo de representación simbólica interna y operativa (Giere, 1992). Considerando que los modelos científicos y didácticos son representaciones del mundo, con su propia lógica interna, sus relaciones de semejanza con los fenómenos y sus medios expresivos o lenguajes simbólicos especializados. En particular, los modelos didácticos son representaciones de orden superior (Adúriz-Bravo y Morales, 2002). 
La utilización del análisis de videos experimentales permite incorporar con facilidad, en las condiciones escolares, investigaciones del mundo real y operar con aplicaciones auténticas de las representaciones gráficas, numéricas y analíticas en estudios de Física (también de Matemática) de manera económica a pesar de ser una sofisticada tecnología de punta.

De hecho, esta tecnología constituye un fértil punto de partida para la utilización de los recursos informáticos en la solución de problemas de carácter experimental en el proceso de enseñanza aprendizaje de la Física y para transformar los trabajos de laboratorio habituales en verdaderas tareas de interés. Esto está en correspondencia con las amplias posibilidades de utilizar este recurso en muchos de los temas de Física para estudiar problemáticas experimentales reales y complejas de manera interesante y asequible para los estudiantes de preuniversitario.

El análisis semiótico sobre el texto en tracker.

Icono: Diseño del video seleccionado (una vista)

Cualisigno: Posibles diseños de la simulación. (Planificación) Modelo didáctico seleccionado para ser simulado. Posibles diseños de la simulación. (Planificación) Modelos (físicos y didácticos) disponibles.

Icono: Diseño del video (una vista) Cualidades formales seleccionadas. (Detalles del diseño de la vista: los elementos dibujados y sus propiedades (forma, color, tamaño, posición en la pantalla, etc.) Vista o diagrama del diseño de la simulación. El valor del diseño en función de las características que comparten el objeto simulado.

Rema: Eficiencia representativa del diseño. La representación gráfica. Valor expresivo de los rasgos utilizados. Cómo se relaciona el objeto firmado con las variables que lo caracterizan. Cómo es esta relación en función de lo que la herramienta permite. Eficacia representativa del diseño dibujado. Valor diferencial del video elegido. (Ventajas con respecto a otros)

Indice: La simulación del fenómeno. (La simulación en ejecución). SINSIGNO: El Programa (Tracker) que se ejecute la simulación. Las propiedades de los objetos del fenómeno (variables y elementos de la vista) en tiempo de ejecución, definidas por las distintas instancias de tiempo y por las relaciones que se definen entre ellas. El programa que ejecuta la simulación. La evolución de la simulación en el tiempo. Condiciones o restricciones que determinan la dinámica de la simulación. La simulación del fenómeno. Los objetos de la simulación. Aquellos relacionados directamente con la vista del fenómeno y los que permiten la interacción o el control de la misma. Estos últimos pueden ser de ejecución, de visualización o de modificación de parámetros o variables. Modelo creado o Video real. La simulación del fenómeno. Una ejecución de la simulación. 
Dicisigno: El valor de la simulación como herramienta que permite representar el modelo del fenómeno. El valor atribuido a la forma de cada objeto de la simulación (tamaños, colores, proporciones, posiciones, etc.) en función del fenómeno físico. El valor de la simulación como herramienta que permite representar el modelo del fenómeno. El valor de la interacción como medio para actuar sobre la simulación.

Símbolo: La interpretación que logran los estudiantes sobre el fenómeno representado.

Legisigno: Las posibles interpretaciones de los estudiantes. Distintas posibilidades de relaciones interpretativas. Las posibles interpretaciones de los estudiantes. El nivel de interpretación posible para los alumnos respecto al fenómeno analizado. La interpretación efectivamente producida en la mente de los estudiantes sobre el fenómeno simulado. Cada una de las relaciones interpretativas efectivamente establecidas. La interpretación efectivamente producida en la mente de los alumnos sobre el fenómeno simulado.

Argumento: El valor argumentativo que tiene la interpretación del fenómeno para el estudiante. El valor cognitivo de las relaciones interpretativas. El valor argumentativo que tiene la interpretación para el alumno El desarrollo de habilidades interpretativas precedentes acerca del fenómeno. Lo que puede explicar hasta donde comprendió el fenómeno.

\section{Conclusiones.}

- El uso de la tecnología ha generado una nueva manera de creer en el futuro. La misma se asienta como condicionante del universo cultural. El uso de los recursos informáticos como tecnología clave de finales de este siglo, ha generado nuevas manifestaciones, nuevos significados y redefiniciones de viejos términos.

- La utilización del análisis de videos experimentales en las clases de Física, permite incorporar con facilidad, en las condiciones escolares, investigaciones del mundo real y operar con aplicaciones auténticas de las representaciones gráficas, numéricas y analíticas en estudios relacionados con la vida práctica de manera económica a pesar de ser una sofisticada tecnología de punta.

\section{Referencias bibliográficas.}

Adúriz-Bravo, A. Morales, L. (2002). El concepto de modelo en la enseñanza de la Física. Consideraciones epistemológicas, didácticas y retóricas. Cad. Brás. Ens. Fís.Vol.19(1), pp.79-92.

Brown, D. (2009). Tracker 2.54. Recuperado de www.cabrillo.edu/ dbrown/tracker

Eco, U. (1986). La Estructura Ausente. Introducción a la Semiótica. 3ra. ed. Barcelona: Lumen.

Eco, H. (1980). Tratado de Semiótica. México: Nueva Imagen, pp. 80-81 
Esquembre, F. (2005). Creación de simulaciones interactivas en Java. Aplicaciones a la enseñanza de la Física. Madrid: Pearson Educación S.A.

Ferreira, J., Barr, P. Noble, J. (2005). The Semiotic of User interfaceRedesign, 6th Australasian User Interface Conference, (AUIC2005), Newcastle. Conferences in Research and Practice in Information Technology, Vol. 40. .M. Billinghurst and A. Cockburn. Recuperado de http://crpit.com/confpapers/CRPITV40Ferreira.pdf

Galagovsky, L. Adúriz-Bavo, A. (2001). Modelos y analogías en la enseñanza de las ciencias naturales. El concepto de modelo didáctico analógico. Enseñanza de las Ciencias, Vol. 19.

Giere, R. (1992). La explicación de la Ciencia. Un acercamiento cognoscitivo. México. S/e, p.103

Gil, D., Macedo, B., Martínez, J., Sifredo, C. y Valdés, P. (2005). ¿Cómo promover el interés por la cultura científica? Una propuesta didáctica fundamentada para la educación científica de jóvenes de 15 a 18 años. España: OREALC/UNESCO-Santiago

Magariños, J.A. 1983. El signo. Las fuentes teóricas de la semiología: Saussure, Peirce, Morris. Buenos Aires: Hachette, pp. 2-6

Scolari, C. A. (2004). Hacer clic. Hacia una semiótica de las interacciones digitales, DeSignis Vol. 5, pp. 26-30

Scolari, C A. (2003). La narración interactiva. Interfaces y sintaxis, acciones y pasiones. Revista Iberoamericana de Comunicación, Vol. 4, pp.1-13.

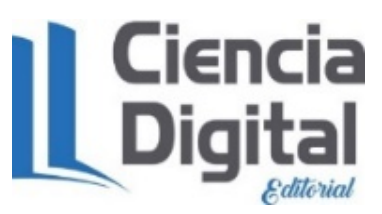




\section{Para citar el artículo indexado}

León Arencibia, A., Rodríguez Brito, B. E., \& Dorvigny González, B. de la C. (2020). Análisis semiótico de videos en la resolución de problemas de la física. AlfaPublicaciones, 1(2), 41-51. https://doi.org/10.33262/ap.v1i2.7

\section{Ciencia \\ Ligital}

El artículo que se publica es de exclusiva responsabilidad de los autores y no necesariamente reflejan el pensamiento de la Revista Alpha Publicaciones.

El artículo queda en propiedad de la revista y, por tanto, su publicación parcial y/o total en otro medio tiene que ser autorizado por el director de la Revista Alpha Publicaciones.
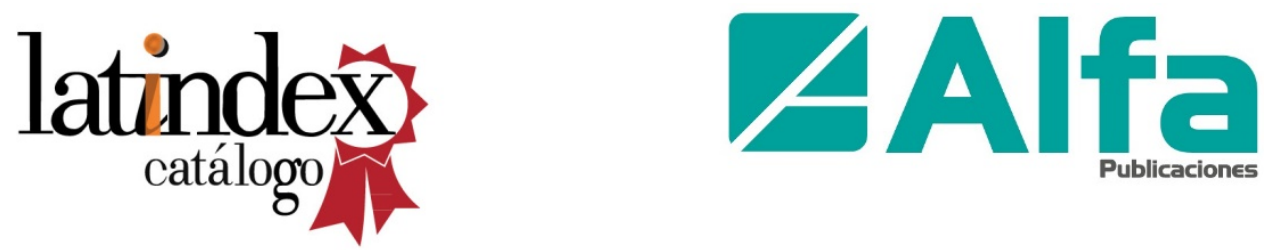